\title{
Ranibizumab Pretreatment in Vitrectomy with Internal Limiting Membrane Peeling on Diabetic Macular Edema in Severe Proliferative Diabetic Retinopathy
}

\author{
Jian Guan · Na Cai · Li-Min Liu • Ning Zhao · Ning-ning Liu
}

Received: February 29, 2020 / Published online: April 30, 2020

(C) The Author(s) 2020

\section{ABSTRACT}

\begin{abstract}
Aim: To evaluate the efficacy of intravitreal ranibizumab (IVR) pretreatment for pars plana vitrectomy (PPV) with internal limiting membrane (ILM) peeling in severe proliferative diabetic retinopathy (PDR) combined with macular edema (ME).
\end{abstract}

Methods: Sixty-three patients with ME and PDR were divided into IVR and control groups. Three days before PPV stripping, ranibizumab was injected into the patients in the IVR group. The patients were followed for 6 months. The bestcorrected visual acuity (BCVA), visual acuity improvement, centre macular thickness (CMT), and intraoperative and postoperative complications were compared between the two groups. Results: The BCVA of the IVR group was significantly improved at 1,3 and 6 months compared with the preoperative BCVA $(P<0.01)$. The BCVA of the control group was significantly

Digital Features To view digital features for this article go to https://doi.org/10.6084/m9.figshare.12136257.

Electronic supplementary material The online version of this article (https://doi.org/10.1007/s13300020-00822-0) contains supplementary material, which is available to authorized users.

J. Guan · N. Cai · L.-M. Liu · N. Zhao · N. Liu (ه) The Department of Ophthalmology, First Affiliated Hospital of China Medical University, Shenyang, Liaoning Province, China

e-mail: ningning1688@126.com improved at 3 and 6 months compared with the preoperative BCVA $(P<0.01)$, but was not significantly improved at 1 month. At 1 and 3 months, the BCVA of the IVR group was significantly better than that of the control group after surgery, with no difference between the two groups at 6 months. The CMT of the IVR group was thinner than that of the control group at 1 and 3 months $(P<0.01)$, with no significant difference at 6 months after surgery. The surgical time, the risk of intraoperative bleeding, the incidence of iatrogenic retinal breaks, the frequency of endodiathermy and the rate of silicone oil tamponade were significantly different between the two groups (all $P<0.05$ ). There was no significant difference between the two groups in terms of postoperative complications.

Conclusions: Ranibizumab pretreatment may improve the outcome of PPV with ILM peeling for severe PDR with ME by decreasing ME and intraoperative complications.

Keywords: Diabetic macular edema; Proliferative diabetic retinopathy; Ranibizumab; Vitrectomy with internal limiting membrane peeling 


\section{Key Summary Points}

Epidemiological studies and clinical observations suggest that the incidence of diabetic macular edema (DME) in patients with severe PDR is up to $70 \%$, which is one of the main causes of visual loss and even blindness in patients with DR

The treatment of DME mainly includes laser photocoagulation, glucocorticoid or anti-VEGF drug intravitreal injection therapy and vitreous surgery. However, pan-retinal photocoagulation (PRP) can result in limited peripheral vision, decreased night version and aggravate diabetic macular edema

Both glucocorticoids and anti-VEGF drugs can rapidly reduce macular edema (ME) and improve the visual function, but they all have a short duration of action and require repeated injections

Consequently, PPV with ILM peeling has been applied in the surgical treatment of fundus diseases, especially in macular hole (MH), ME, macular epiretinal membrane, retinal splitting and other tractive macular diseases. However, the efficacy of vitrectomy combined with internal limiting membrane stripping in the treatment of PDR with DME has rarely been reported

The purpose of this study was to evaluate the efficacy of preoperative intravitreal injection of ranibizumab combined with vitrectomy with internal limiting membrane peeling for macular edema on diabetic macular edema in severe proliferative diabetic retinopathy

\section{INTRODUCTION}

Proliferative diabetic retinopathy (PDR) is a common complication of diabetes mellitus
(DM), often resulting in visual impairment due to serious complications (e.g. neovascularization, vitreous haemorrhage and traction retinal detachment) [1]. The incidence of diabetic macular edema (DME) in patients with severe PDR is up to $70 \%$, and DME is one of the main causes of visual loss and even blindness in patients with DR [2].

The treatment for DME mainly includes laser photocoagulation, glucocorticoid or anti-vascular endothelial growth factor (VEGF) drug intravitreal injection therapy and vitreous surgery [3-5]. However, pan-retinal photocoagulation (PRP) can result in limited peripheral vision, decreased night version and aggravated DME [6]. Some patients with PDR who are treated with laser photocoagulation have poor visual acuity.

Both glucocorticoids and anti-VEGF drugs can rapidly reduce macular edema (ME) by reducing the leakage of vascular permeability and inflammatory factors and improve visual function, but they all have a short duration of action and require repeated injections [5, 7]. Therefore, when DME occurs in late PDR and is accompanied by neovascularization, vitreous haemorrhage, preretinal proliferative membrane and tractive retinal detachment, vitrectomy may be the most effective treatment to prevent further deterioration of the disease. However, simple vitrectomy does not completely eliminate macular edema, and a residual inner limiting membrane (ILM) remains after surgery [8]. Under pathological conditions, the ILM can be used as a scaffold for the proliferation of fibrocytes and pigment cells, it adheres closely to the posterior cortex of the vitreous and then participates in the pathological process of macular diseases such as cystoid macular edema, macular hole (MH) and anterior macular membranes. Peeling away the ILM can not only relax the adhesions of the macula of the vitreous and completely relieve the traction of the posterior boundary membrane of the vitreous on the macula but also reduce the adhesion of VEGF, inflammatory factors and other related vasoactive factors and reduce the recurrence rate of vitreous macular interface disease. Consequently, PPV with ILM peeling has been applied in the surgical treatment of fundus 
diseases, especially in MHs, ME, macular epiretinal membranes, retinal splitting and other tractive macular diseases [9]. The need for combined ILM peeling in patients with DME, however, is currently controversial. A large number of studies have shown that vitrectomy combined with internal membrane peeling is conducive to the recovery of macular edema and improved vision [10]. For simple vitrectomy or vitrectomy with internal limiting membrane peeling, it is impossible to determine the macular state before surgery because of the complexity of the fundus in patients with PDR, such as those with severe vitreous haemorrhage or tractional retinal detachment. This makes vitrectomy more challenging. Intraoperative bleeding often occurs during the process of removing fibrous neovascularization membranes, thus affecting the surgical visual field, increasing intraoperative and postoperative complications and ultimately failing to improve visual acuity [11]. In recent years, a large number of studies have shown that preoperative pretreatment with anti-VEGF drugs can reduce intraoperative bleeding, iatrogenic retinal tears and postoperative early bleeding [12]. Ranibizumab is a human monoclonal antibody against the Fab segment of VEGF that inhibits the activation of the VEGFA subtype. Ranibizumab injection before vitrectomy has been reported as an adjuvant therapy for severe PDR [13]. However, the efficacy of vitrectomy combined with internal limiting membrane stripping in the treatment of PDR with DME has rarely been reported. The purpose of this study was to evaluate the efficacy of preoperative intravitreal injection of ranibizumab combined with vitrectomy with internal limiting membrane peeling for macular edema on diabetic macular edema in severe proliferative diabetic retinopathy.

\section{METHODS}

\section{Study Design}

This was a retrospective, comparative and nonrandomized study, in line with the principles of the Helsinki Declaration, and was approved by the Ethics Committee of the First Affiliated Hospital of Shenyang, China Medical University. We reviewed the medical notes of 63 patients with PDR who underwent vitrectomy with or without pretreatment as a result of nonabsorbent VH between January 2013 and December 2015 and who completed at least 6 months of follow-up. Patients were divided into the IVR group and the control group according to whether an intravitreal injection of ranibizumab (IVR) was administered before surgery. In the control group, 32 patients received vitrectomy only from January 2013 to December 2014; 31 patients in the IVR group underwent vitrectomy from January 2015 to December 2015. Pre-IVR pretreatment, all participants signed an informed consent form explaining the benefits and risks prior to surgery.

\section{Participants}

All patients underwent a comprehensive examination with the best-corrected visual acuity (BCVA), intraocular pressure (IOP), B-ultrasound, slit lamp biomicroscopy and 90D NonContact Slit Lamp Lens. During follow-up, macular edema was measured by fundus photography, optical coherence tomography and fluorescein angiography (FFA).

The inclusion criteria were as follows: (1) patients with PDR and moderate vitreous haemorrhage (only disc faintly visible) and severe haemorrhage (no fundus view) was selected as the study object; (2) patients with type 2 diabetes aged at least 50 years old (according to the American Diabetes Association or World Health Organization guidelines) and preoperative haemoglobin A1c at most 10\%; (3) non-clearing vitreous haemorrhage within 1 month secondary to PDR; (4) the diagnosis of macular edema dependent on the presence of apparent retinal thickening or lipids in the posterior pole seen by the surgeon during vitrectomy. The severity was classified on the basis of the International Clinical Diabetic Retinopathy and Diabetic Macular Edema Disease Severity Scales [14]: mild-partial retinal thickening or hard posterior pole exudation but 
away from the centre of the macula; moderateretinal thickening or hard exudation close to the centre of the macula but not involved in the centre; and severe-thickening of the retina or hard exudate involving the centre of the macula; (5) during the follow-up period, the DME severity grading standard was examined by fundus photography and FFA: (1) no obvious DME-no obvious retinal thickening or hard exudation at the posterior pole; (2) mild DMEthickening or hard exudation of the retina in the posterior pole away from the centre of the macula; (3) moderate DME-retinal thickening or hard exudation near but not involving the macular centre; and (4) severe DME-retinal thickening or hard exudation involving the macular centre. The exclusion criteria included (1) history of vitrectomy, other intraocular surgery and ocular trauma; (2) complete PVD and an anterior macular membrane; (3) retinal detachment traction (tractive, rhegmatogenous or exudative), macular disease secondary to the outer membrane of the retina, retinal vein occlusion, optic neuropathy and other serious eye diseases; (4) history of laser or anti-VEGF injection; (5) DM accompanied by severe cardiovascular and cerebrovascular diseases, abnormal liver or kidney function and other systemic diseases; (6) coexisting ocular disease caused by macular edema (e.g. branch vein occlusion or central retinal occlusion); and (7) follow-up period less than 6 months.

\section{Treatment Protocol}

One surgeon (Na Cai) with over 20 years of experience in posterior segment surgery performed all injections and surgeries. Patients in the IVR group were intravitreally injected with ranibizumab $(0.5 \mathrm{mg} / 0.05 \mathrm{ml}) 3$ days before PPV and stripped. Ranibizumab was aspirated into a 1 -ml syringe and injected into the vitreous cavity through a 30-gauge needle [15]. All patients underwent standard three-hole 20-gauge pars plana vitrectomy (PPV). The procedures included the removal of the anterior and central vitreous and the vitreous cortex; detachment of the posterior hyaloid created by suction with the vitrectomy instrument; the removal of proliferative membranes with intraocular forceps; endodiathermy or increased infusion pressure used to stop intraoperative bleeding; and ILM peeling performed with ILM forceps by using a continuous curvilinear capsulorhexis technique without staining of the ILM. The ILM was moved and extended approximately 6-disc diameters horizontally and from the upper arcade to the lower arcade vertically around the fovea. All patients underwent intra-retinal laser photocoagulation. All phacoemulsification procedures were performed with phacoemulsification, and intraocular lenses were implanted to avoid the development of cataracts. Finally, $\mathrm{C}_{3} \mathrm{~F}_{8}$ gas or silicone oil ( $\mathrm{SO}$ ) was used when needed at the end of the operation. Subconjunctival injections of dexamethasone $(2 \mathrm{mg})$, topical antibiotics and corticosteroid drops were administered postoperatively.

\section{Study Assessment}

The primary outcome measures after vitrectomy included the BCVA, comparison of visual acuity improvement, and CMT. The secondary outcome measures included intraoperative bleeding, required internal diathermy frequency, need for tamponade, operative time, number of iatrogenic retinal breaks, and postoperative complications.

To compare the similarities between the two groups, we scored vitreous haemorrhage from 0 to 3 [16], (0-none, clear field of view; 1 -mild, visible fundus details but difficult to assess retinal nerve fibre layers or small blood vessels; 2-moderate, only the intervertebral disc was faintly visible; and 3-severe, fundus details were invisible). The severity of intraoperative bleeding was defined as follows: mild bleeding, spontaneous or stopped with a litre bottle; severe bleeding, covering half or more of the posterior pole, required intra-arterial diathermy treatment [17]. We considered recurrent vitreous haemorrhage $(\mathrm{VH})$ as grade 1 or higher $\mathrm{VH}$ occurring more than 1 week after surgery. If the incidence rate was 4 weeks or less, it was considered early recurrent $\mathrm{VH}$; otherwise, it was considered late recurrent $\mathrm{VH}$ [18]. 


\section{Statistical Analysis}

The baseline characteristics of patients were collected and analysed using SPSS version 22 (IBM, Armonk, NY, USA). The Snellen visual acuity was converted to logarithm of the minimum angle of resolution (logMAR) units for statistical analyses. Student's $t$ test was used for pairwise comparisons of age, duration of diabetes, BCVA, IOP, CMT and mean surgical time between the two groups. The results are presented as the mean and standard deviation. The chi-square test or Fisher's exact test was used to analyse differences in sex, intraoperative bleeding, the incidence of iatrogenic retinal breaks, the use of endodiathermy and SO endotamponade, vitreous haemorrhage score and grade of ME. One-way analysis of variance (ANOVA) was used to evaluate BCVA at followup and baseline stages. $P<0.05$ was set for statistical significance. Improvement or reduction in BCVA was defined as two or more change lines; otherwise, BCVA was defined as unchanged. The Mann-Whitney $U$ test was used to determine whether there was a significant difference in visual improvement between the two groups.

\section{RESULTS}

\section{Basic Characteristics}

The characteristics of the patients are shown in Table 1. Sixty-three eyes from 63 patients were studied. There was no significant difference in sex, age, duration of diabetes, preoperative BCVA, intraocular pressure, vitreous haemorrhage score or grade of ME between the groups.

\section{Main Results}

The logMAR BCVA levels were analysed during the follow-up. In the IVR group, the logMAR BCVA level 1 month, 3 months and 6 months after surgery was higher than that before surgery $(P<0.01)$. The logMAR BCVA of the control group was more significantly improved at 3 and 6 months after surgery than that before
Table 1 Baseline characteristics of patients

\begin{tabular}{|c|c|c|c|}
\hline & $\begin{array}{l}\text { Control } \\
\text { group } \\
(n=32)\end{array}$ & $\begin{array}{l}\text { IVR } \\
\text { group } \\
(n=31)\end{array}$ & $P$ value \\
\hline \multicolumn{4}{|l|}{ Sex } \\
\hline Male (\%) & $15(46.87 \%)$ & $15(48.39 \%)$ & \multirow[t]{2}{*}{0.90} \\
\hline Female (\%) & $17(53.13 \%)$ & $16(51.61 \%)$ & \\
\hline \multicolumn{4}{|l|}{ Age ( years) } \\
\hline Mean (SD) & $59.47 \pm 3.56$ & $58.87 \pm 3.02$ & 0.48 \\
\hline \multicolumn{4}{|c|}{ Duration of diabetes (years) } \\
\hline Mean (SD) & $16.16 \pm 2.40$ & $16.48 \pm 2.19$ & 0.57 \\
\hline \multicolumn{4}{|c|}{ Mean BCVA (logMAR) } \\
\hline Mean (SD) & $1.52 \pm 0.51$ & $1.56 \pm 0.62$ & 0.76 \\
\hline \multicolumn{4}{|l|}{ IOP (mmHg) } \\
\hline Mean (SD) & $15.69 \pm 2.09$ & $16.65 \pm 2.50$ & 0.10 \\
\hline \multicolumn{3}{|c|}{ Vitreous haemorrhage score } & 0.71 \\
\hline Modern & $18(56.25 \%)$ & $16(51.61 \%)$ & \\
\hline Sever & $14(43.75 \%)$ & $15(48.39 \%)$ & \\
\hline \multicolumn{3}{|c|}{ Grade of macular edema } & 0.88 \\
\hline Mild & $3(9.38 \%)$ & $2(6.45 \%)$ & \\
\hline Moderate & $15(46.88 \%)$ & $16(51.61 \%)$ & \\
\hline Sever & $14(43.75 \%)$ & 13 (41.94\%) & \\
\hline
\end{tabular}

surgery $(P<0.01)$. However, there was no statistically significant difference at 1 month compared with the preoperative BCVA. At 1 month and 3 months after surgery, the logMAR BCVA of the IVR group was superior to that of the control group $(t=10.94, t=7.93$, $P<0.001)$. There was no significant difference in logMAR BCVA between the two groups 6 months postoperatively $(t=1.32, \quad P>0.05)$ (Table 2).

Table 3 shows visual acuity improvement after operation in the two groups. There was a significant difference in visual acuity improvement between the two groups. At 6 months after surgery, nine patients $(28.13 \%)$ had 
Table2 Change in logMAR BCVA between two groups $(\bar{x} \pm s)$

\begin{tabular}{llll}
\hline & Control group $(\boldsymbol{n}=32)$ & IVR group $(\boldsymbol{n}=31)$ & $P$ \\
\hline Preoperative & $1.52 \pm 0.51$ & $1.56 \pm 0.62$ & $>0.05$ \\
1 month follow-up & $1.43 \pm 0.41$ & $0.59 \pm 0.11^{*}$ & $<0.001$ \\
3 months follow-up & $0.58 \pm 0.07^{*}$ & $0.40 \pm 0.11^{*}$ & $<0.001$ \\
6 months follow-up & $0.41 \pm 0.10^{*}$ & $0.38 \pm 0.13^{*}$ & $>0.05$ \\
\hline
\end{tabular}

*Standing for the comparison of every group with its value before operation $P<0.01$

Table 3 Postoperative visual acuity improvement between the two groups (eye \%)

\begin{tabular}{lcc}
\hline IogMAR BCVA & Control group $(\boldsymbol{n}=\mathbf{3 2})$ & IVR group $(\boldsymbol{n}=\mathbf{3 1})$ \\
\hline Improved & $9(28.13 \%)$ & $21(67.74 \%)$ \\
No change & $21(65.63 \%)$ & $10(32.26 \%)$ \\
Decreased & $2(6.25 \%)$ & $0(0.0 \%)$ \\
\hline
\end{tabular}

improved visual acuity, two had decreased visual acuity (6.25\%) and 21 (65.63\%) had no significant change in visual acuity in the control group. In the IVR group, 21 (67.74\%) patients had improved visual acuity, 10 of 31 eyes $(32.26 \%)$ had no change, and the visual acuity did not decrease $\left(\chi^{2}=10.69, P<0.01\right)$.

The average CMT of the IVR group was significantly lower than that of the control group 1 month and 3 months after the operation $(t=5.60, t=6.15, P<0.01)$. There was no significant difference between the two groups in the mean CMT 6 months after the operation $(t=0.66, P>0.05)$ (Table 4$)$.

Table 5 summarizes the secondary outcomes of the procedure. Endothermy treatment was used in two eyes (6.45\%) in the IVR group. However, the incidence of intraoperative bleeding was $12(37.50 \%)$ in the control group, and 12 eyes (37.50\%) required endovascular treatment. There were significant differences between the two groups in the severity of bleeding control and the use of endothermia $\left(\chi^{2}=5.03, P<0.05, \chi^{2}=8.78, P<0.01\right)$. There were 21 cases $(65.63 \%)$ of iatrogenic retinal rupture in the control group and three cases $(9.68 \%)$ of iatrogenic retinal rupture in the IVR group, the difference was statistically significant $\left(\chi^{2}=20.90, P<0.001\right)$. In addition, the rate of silicone oil tamponade in the IVR group $(12.90 \%)$ was lower than that in the control group (40.63\%), the difference was statistically significant $\left(\chi^{2}=6.14, P<0.05\right)$. The mean total surgical time was shorter in the IVR group $(92.60 \pm 9.65 \mathrm{~min})$ than in the control group (135.41 $\pm 42.39 \mathrm{~min})(t=5.53, P<0.01)$. These results suggest that intravitreal ranibizumab pretreatment for PPV in patients with PDR reduces the difficulty of the operation and the incidence of intraoperative complications [19].

\section{Postoperative Complications and Adverse Events}

During the follow-up period, transient intraocular hypertension occurred in three eyes $(9.38 \%)$ of 32 eyes in the control group. Among the 31 eyes in the IVR group, transient intraocular hypertension (9.68\%) occurred in three eyes. Intraocular pressure (IOP) elevation was defined as intraocular pressure greater than $25 \mathrm{mmHg}$ on the first postoperative day. If the IOP was elevated, the subject was observed until the IOP dropped to $25 \mathrm{mmHg}$ or lower. Three 
Table 4 Postoperative CMT findings between two groups

\begin{tabular}{llll}
\hline & Control group $(\boldsymbol{n}=\mathbf{3 2})$ & IVR group $(\boldsymbol{n}=\mathbf{3 1})$ & $\boldsymbol{P}$ \\
\hline 1 month follow-up & $380.84 \pm 75.65$ & $289.68 \pm 50.73$ & $<0.01$ \\
3 months follow-up & $335.06 \pm 53.57$ & $266.23 \pm 32.33$ & $<0.01$ \\
6 months follow-up & $260.50 \pm 27.81$ & $255.71 \pm 30.17$ & $>0.05$ \\
\hline
\end{tabular}

Table 5 Intraoperative parameters between two groups

\begin{tabular}{llll}
\hline Parameters & Control group $(\boldsymbol{n}=\mathbf{3 2})$ & IVR group $(\boldsymbol{n}=\mathbf{3 1})$ & $\boldsymbol{P}$ \\
\hline Duration of vitrectomy & & & \\
Mean (SD) & $135.41 \pm 42.39$ & $92.60 \pm 9.65$ & $<0.01$ \\
Intraoperative bleeding & $12(37.50 \%)$ & $4(12.90 \%)$ & $<0.05$ \\
Iatrogenic retinal breaks & $21(65.63 \%)$ & $3(9.68 \%)$ & $<0.001$ \\
Endodiathermy applications 12(37.50\%) & $12(37.50 \%)$ & $2(6.45 \%)$ & $<0.01$ \\
Silicone oil tamponade & $13(40.63 \%)$ & $4(12.90 \%)$ & $<0.05$ \\
\hline
\end{tabular}

patients received IOP-lowering drugs. IOP in all patients fell to the normal range within 1 week. Mild recurrent $\mathrm{VH}$ with visible fundus details but with difficulty evaluating the retina nerve fibre layer or small vessels occurred in two eyes $(6.25 \%)$ in the control group and one eye (3.23\%) in the IVR group at the 2-week followup. Patients were required to control blood sugar and orally take Chinese medicine (He Xue Ming $\mathrm{Mu}$ tablets). After 2 weeks, vitreous haemorrhage was completely absorbed. At the end of follow-up, no recurrence of macular epiretinal membrane, $\mathrm{ME}$, serious complications, endophthalmitis or allergic reactions was observed in either group. There was no significant difference in transient IOP or vitreous haemorrhage between the two groups.

\section{DISCUSSION}

Studies have confirmed the efficacy of vitrectomy with ILM peeling in the treatment of DME $[20,21]$. However, whether the removal of the ILM can resolve macular edema and improve vision remains controversial. Yamamoto et al. revealed that ILM peeling did not reduce macular edema [8]. A study investigated 42 cases of non-tractional DME and found that vitrectomy with ILM peeling promoted the absorption of tractive macular edema and improved VA [22]. Our data demonstrated that BCVA was significantly higher in the control group and the IVR group than in the preoperative period, especially 3 months and 6 months after surgery. Vitrectomy combined with ILM improved visual acuity in patients with severe PDR with macular edema, consistent with previous studies. However, BCVA was not statistically significant 1 month after vitrectomy in the control group. One reason for this result may be that inflammation in patients with diabetes mellitus is more evident than that in normal people, affecting the absorption of retinal edema. Inflammatory exudation can also aggravate the opacity of the refractive interstitium. On the other hand, silicone oil tamponade in the vitreous cavity in the control group was greater than that in the IVR group. The effect of silicone oil refractive factors may be another cause 
of poor visual acuity in the early postoperative period. In contrast, the IVR group demonstrated more significant visual acuity improvements at 1 and 3 months; however, the improvement at 6 months between the two groups was not statistically significant. Some cytokines, including inflammatory cytokines, are involved in the pathogenesis of diabetic macular edema. Intraocular cytokines and VEGF levels are elevated in patients with DME and proliferative DR. VEGF inhibition improves vision and reduces macular edema in patients with DME $[23,24]$. In the IVR group, preoperative intravitreal injection of ranibizumab pretreatment reduced intraocular VEGF levels and various inflammatory cytokine concentrations. The half-life of ranibizumab is estimated to be 9 days in the vitreous [25]. Although the majority of ranibizumab in the pretreated vitreous may be removed with vitrectomy, the remaining retina may still be functional [26]. Local factors partly contributed to the improvements in VA and DME in the early stage after surgery. In addition, VEGFR2 had anti-neovascularization activity, and the expression of VEGFR2 increased after ranibizumab treatment. The upregulation of VEGFR2 may contribute to the inhibition of macular edema by anti-vascular endothelial growth factor [27]. This is in accordance with the improvement in VA and the decrease in macular edema in the IVR group compared with the control group 1 and 3 months after surgery. CMT in the IVR group was significantly different from that in the control group 1 and 3 months after surgery and then decreased slowly. The trend of change was consistent with that of BCVA after the operation. At the last follow-up of 6 months, the results showed that the improvement in VA in the IVR group was significantly better than that in the control group. We consider the following mechanisms explaining the difference in visual rehabilitation between the two groups: preoperative pretreatment with ranibizumab in the IVR group could produce most of the retinal neovascularization atrophy, and neovascularization membrane fibrosis atrophy in the fundus retina and in front of the optic disc, effectively reducing the amount of bleeding and the number of electrocoagulation events.
Patients with PDR and traction retinal detachment undergo PPV surgery because the retina is extremely thin, and iatrogenic holes easily appear when the proliferative membrane is removed. However, intravitreal injection of ranibizumab can reduce the permeability of blood vessels, reduce the edema of the retina and enhance the anti-pulling ability of the retina to a certain extent. At the same time, the proliferative membrane becomes thinner, the membrane tissue becomes layered and loose, and the adhesion becomes poor between the proliferative membrane and the retina, which reduces the difficulty of dissociation of the proliferative membrane and reduces the generation of iatrogenic holes. Intravitreal injection of ranibizumab can decrease the bleeding rate during the operation, which can provide a clear visual field during the operation, increase the visibility of the retina, simplify the operation, reduce the difficulty of the operation and greatly shorten the operation time. Surgical operation or surgical trauma may affect macular physiological function. It may play a role in reducing the effect of diabetic macular edema. Many researchers have shown that IVR can decrease diabetic macular edema and improve visual acuity [28].

The limitations of our study are its retrospective nature, the small sample number and the short follow-up period. Studies with larger sample sizes are required to further evaluate the efficacy and safety of IVR treatment in vitrectomy with ILM peeling in severe PDR combined with DME in the longer term.

\section{CONCLUSIONS}

IVR pretreatment before vitrectomy with ILM peeling and simple vitrectomy with ILM peeling can improve visual function of eyes with severe PDR macular edema. However, IVR treatment before vitrectomy with ILM peeling is more effective in improving VA, especially in visual rehabilitation after surgery by reducing macular edema, and reducing intraoperative and postoperative complications than vitrectomy with ILM peeling alone. It is an effective method for treating severe PDR with macular edema. 


\section{ACKNOWLEDGEMENTS}

We thank all those who participated in the study. We thank Jing-nan Han, Shi-qiao Jia, Jin Geng, and other doctors for their assistance in ophthalmic examinations.

Funding. No funding or sponsorship was received for this study or publication of this article. The Rapid Service Fee was funded by the authors.

Authorship. All the authors are in line with the International Committee of Medical Journal Editors (ICMJE) standards for the author's qualification, responsible for the integrity of the entire work, and have approved the publication of this edition.

Disclosures. Jian Guan, Na Cai, Li-Min Liu, Ning Zhao and Ning-ning Liu have nothing to disclose.

Compliance with Ethics Guidelines. This study was approved by the Ethics Committee of the First Affiliated Hospital of Shenyang, China Medical University. All procedures implemented in studies involving human participants are in accordance with the ethical standards of the agency and/or the National Research Council, the 1964 Helsinki Declaration and subsequent amendments or similar ethical standards. Informed consent was obtained for all individuals involved in the study.

Data Availability. Data sets generated and/ or analyzed in the current research process may be obtained from the appropriate authors, as long as reasonably required.

Open Access. This article is licensed under a Creative Commons Attribution-NonCommercial 4.0 International License, which permits any non-commercial use, sharing, adaptation, distribution and reproduction in any medium or format, as long as you give appropriate credit to the original author(s) and the source, provide a link to the Creative Commons licence, and indicate if changes were made. The images or other third party material in this article are included in the article's Creative Commons licence, unless indicated otherwise in a credit line to the material. If material is not included in the article's Creative Commons licence and your intended use is not permitted by statutory regulation or exceeds the permitted use, you will need to obtain permission directly from the copyright holder. To view a copy of this licence, visit http://creativecommons.org/licenses/by$\mathrm{nc} / 4.0 /$.

\section{REFERENCES}

1. Zhong J, Jia J, Yu J, Zhang L, Xiang Y. Preoperative photocoagulation reduces corneal endothelial cell damage after vitrectomy in patients with proliferative diabetic retinopathy. Medicine (Baltimore). 2017;96:e7971.

2. Bandello F, Battaglia Parodi M, Lanzetta P, et al. Diabetic macular edema. Dev Ophthalmol. 2017;58:102-38.

3. Qiao G, Dong WJ, Dai Y, Jiang ZH, Guo HK. Diabetic macular edema in proliferative stage treated with anti-vascular endothelial growth factor agent and triamcinolone acetonide by laser-based strategies. Int J Ophthalmol. 2017;18(10):1113-9.

4. Gungel H, Altan C, Baylancicek DO, Karagoz Y, Kisbet $\mathrm{T}$. The effects of 23-gauge pars plana vitrectomy on orbital circulation using Doppler ultrasonography in diabetic macular edema with epiretinal membrane and taut posterior hyaloid. Curr Eye Res. 2017;42:118-24.

5. Muston D, Korobelnik JF, Reason T, et al. An efficacy comparison of anti-vascular growth factor agents and laser photocoagulation in diabetic macular edema: a network meta-analysis incorporating individual patient-level data. BMC Ophthalmol. 2018;18:340.

6. Lee CM, Olk RJ. Modified grid laser photocoagulation for diffuse diabetic macular edema. Long-term visual results. Ophthalmology. 1991;98:1594-602.

7. Martidis A, Duker JS, Greenberg PB, et al. Intravitreal triamcinolone for refractory diabetic macular edema. Ophthalmology. 2002;109:920-7.

8. Yamamoto T, Hitani K, Sato Y, Yamashita $H$, Takeuchi S. Vitrectomy for diabetic macular edema with and without internal limiting membrane removal. Ophthalmologica. 2005;219:206-13. 
9. Hong N, Huang BS, Tong JP. Primary silicone oil tamponade and internal limiting membrane peeling for retinal detachment due to macular hole in highly myopic eyes with chorioretinal atrophy. BMC Ophthalmol. 2015;15:165.

10. Iglicki M, Lavaque A, Ozimek M, et al. Biomarkers and predictors for functional and anatomic outcomes for small gauge pars planavitrectomy and peeling of the internal limiting membrane in naïve diabetic macular edema: The VITAL Study. PLoS One. 2018;13:e0200365.

11. Nicholson BP, Schachat AP. A review of clinical trials of anti-VEGF agents for diabetic retinopathy. Graefes Arch Clin Exp Ophthalmol. 2010;248(7): 915-30.

12. Faisal SM, Tahir MA, Cheema AM, Anjum MI. Pars plana vitrectomy in vitreous hemorrhage with or without intravitreal bevacizumab: a comparative overview. Pak J Med Sci. 2018;34:221-5.

13. Yang KB, Zhang H, Li SJ, et al. Conbercept and ranibizumab pretreatments in vitrectomy with silicone oil infusion for severe diabetic retinopathy. J Ocul Pharmacol Ther. 2019;35(3):161-7.

14. Wilkinson CP, Ferris FL III, Klein RE, et al. Proposed international clinical diabetic retinopathy and diabetic macular edema disease severity scales. Ophthalmology. 2003;110(9):1677-82.

15. Dong F, Yu C, Ding H, Shen L, Lou D. Evaluation of intravitreal ranibizumab on the surgical outcome for diabetic retinopathy with tractional retinal detachment. Medicine (Baltimore). 2016;95:e2731.

16. Ahn J, Woo SJ, Chung H, Park KH. The effect of adjunctive intravitreal bevacizumab for preventing postvitrectomy hemorrhage in proliferative diabetic retinopathy. Ophthalmology. 2011;118: 2218-26.

17. Mao JB, Wu HF, Chen YQ, et al. Effect of intravitreal conbercept treatment before vitrectomy in proliferative diabetic retinopathy. Int J Ophthalmol. 2018;11:1217-21.

18. Yang $\mathrm{X}, \mathrm{Xu} \mathrm{J}$, Wang $\mathrm{R}$, et al. A randomized controlled trial of conbercept pretreatment before vitrectomy in proliferative diabetic retinopathy. J Ophthalmol. 2016;2016:2473234.

19. Pakzad-Vaezi K, Albiani DA, Kirker AW, et al. Randomized study comparing the efficacy of bevacizumab and ranibizumab as pre-treatment for pars plana vitrectomy in proliferative diabetic retinopathy. Ophthalmic Surg Lasers Imaging Retina. 2014;45(6):521-4.

20. Iglicki $\mathrm{M}$, Lavaque A, Ozimek $\mathrm{M}$, et al. Biomarkers and predictors for functional and anatomic outcomes for small gauge pars plana vitrectomy and peeling of the internal limiting membrane in naïve diabetic macular edema: The VITAL Study. PLoS One. 2018;13(7):e0200365.

21. Kim YM, Chung EJ, Byeon SH, Lee SC, Kwon OW, Koh HJ. Pars plana vitrectomy with internal limiting membrane peeling compared with intravitreal triamcinolone injection in the treatment of diabetic macular edema. Ophthalmologica. 2009;223: $17-23$.

22. Ulrich JN. Pars plana vitrectomy with internal limiting membrane peeling for nontractional diabetic macular edema. Open Ophthalmol J. 2017;11: 5-10.

23. Kocabora MS, Telli ME, Fazil K, et al. Serum and aqueous concentrations of inflammatory markers in diabetic macular edema. Ocul Immunol Inflamm. 2016;24:549-54.

24. Diabetic Retinopathy Clinical Research Network, Elman MJ, Qin $\mathrm{H}$, et al. Intravitreal ranibizumab for diabetic macular edema with prompt versus deferred laser treatment: three-year randomized trial results. Ophthalmology. 2012;119:2312-8.

25. $\mathrm{Xu} \mathrm{L}, \mathrm{Lu} \mathrm{T}$, Tuomi $\mathrm{L}$, et al. Pharmacokinetics of ranibizumab in patients with neovascular age-related macular degeneration: a population approach. Invest Ophthalmol Vis Sci. 2013;54:1616-24.

26. Zhao LQ, Zhu $\mathrm{H}$, Zhao PQ, Hu YQ. A systematic review and meta-analysis of clinical outcomes of vitrectomy with or without intravitreal bevacizumab pretreatment for severe diabetic retinopathy. Br J Ophthalmol. 2011;95:1216-22.

27. Lim SW, Bandala-Sanchez E, Kolic M, et al. The influence of intravitreal ranibizumab on inflammation-associated cytokine concentrations in eyes with diabetic macular edema. Invest Ophthalmol Vis Sci. 2018;59:5382-90.

28. Diabetic Retinopathy Clinical Research Network, Scott IU, Edwards AR, et al. A phase II randomized clinical trial of intravitreal bevacizumab for diabetic macular edema. Ophthalmology. 2007;114:1860-7. 